\title{
DEVELOPMENT OF ROBOT MANIPULATOR FOR LAPAROSCOPIC SURGERY WITH FORCE DISPLAY USING PNEUMATIC SERVO SYSTEM
}

\author{
Kotaro TADANO*, Kenji KAWASHIMA* and Toshiharu KAGAWA* \\ * Precision and Intelligence Laboratory \\ Tokyo Institute of Technology \\ 4259 R2-46 Midori-ku, Nagatsuta, Yokohama, 226-8503 Japan \\ (E-mail: tadano@k-k.pi.titech.ac.jp)
}

\begin{abstract}
In the teleoperated minimally invasive surgery systems, measurement and display of sense of force to the operator is a problem In this paper, we have developed a master-slave system having 7-DOFs for laparoscopic surgery, which can provide force feedback to the surgeon without using force sensors at the slave manipulator. Pneumatic actuators are used for the slave manipulation because they are effective for haptic devices due to the facility in measurement and control of their driving force. A control method to compensate the effect of the pipeline between the control valves and the actuators is proposed and applied. Then, an impedance control is applied to the slave manipulator and an admittance control is applied for the master manipulator. Finally, a bilateral control is achieved by combining the controllers. Experimental results show that the developed system successfully display the contact force on the slave side to the operator on the master side.
\end{abstract}

\section{KEY WORDS}

Pneumatics, Surgical robot, Laparoscopic surgery, Master-slave system, Bilateral control

\section{INTRODUCTION}

As alternative method to open surgery, minimally invasive laparoscopic surgery is an effective method. Robotic manipulators, which have multi-DOFs at their tip, have been reported as alternative to conventional instruments to support the surgery [1][2].

A master-slave type manipulator has advantages that the intuitiveness could be realized. A surgeon is able to teleoperate the forceps at the master side, as if he/she handles the forceps at the slave side in the abdominal cavity[3]. In this type, however, has the problem that the sense of force is lost. In order to realize safer and more precise operation using robotic manipulator especially master-slave system, force measurement and feedback are very important [4].
To realize a precise position control of the manipulator, using electric motors with high reduction gears is effective while the back drivability is small. Sensing the accurate force with such manipulators needs a force sensor at near the tip. However, the sensor at the end of forceps manipulator makes sterilizing and downsizing difficult.

In this research, we have developed a master-slave system with 7-DOFs forceps manipulators that is able to provide a force display to surgeons without a force sensor. To achieve this, we use pneumatic actuators, because they are effective for a haptic device due to the facility in measurement and control of their driving force, and enable the estimation of the external force from the driving force and the impedance. A control method to compensate the effect of the pipeline between 
the valves and the actuators is proposed and applied. Then, an impedance control is applied with the compensator to the slave side. For the master manipulator we have developed, an admittance control is used. Finally, a bilateral control is achieved.

\section{DEVELOPED 7DOFS SLAVE MANIPULATOR}

Fig. 1 shows the developed forceps manipulator that has 7-DOFs. The manipulator consists of a tip and a supporting part. The supporting part was introduced in the former paper, which feature is compact and the pivot point at the trocar cannula is immovable mechanically without direct support [5].

The tip part of the developed manipulator has a roll, two bending joints and a rotating movement can be achieved. The motions are transmitted using wire ropes. The diameter of the manipulator is $10 \mathrm{~mm}$ that is useful for laparoscope surgery.

The feature of this forceps is that the bending and gripper were realized at the same joint. Therefore, the tip part becam compact. The rotary actuator with low friction (KURODA, PRNAJ1D-90-45) is used. The move angle is more than 73 degree for each joint and the maximum torque is $208 \mathrm{Nm}$. The driving force of the actuator is given as

$$
\boldsymbol{F}_{\mathrm{dr}}=A_{1} P_{1}-A_{2} P_{2}
$$

where $A$ and $P$ indicate the pressurized area and the differential pressure and the index 1 and 2 shows the room of the pneumatic cylinder. The supply pressure was set at $500 \mathrm{kPa}$ and a pressure sensor having a resolution of $20 \mathrm{~Pa}$ was used. The actuator was controlled by a five ports servo valve made by FESTO. The servo valve receives the voltage signal and controls the flow rate to the actuator.

Pressures in the actuator were measured with semiconductor type sensors installed at the out port of the servo valve. This is because to make the forceps manipulator as possible as free from sensors to make

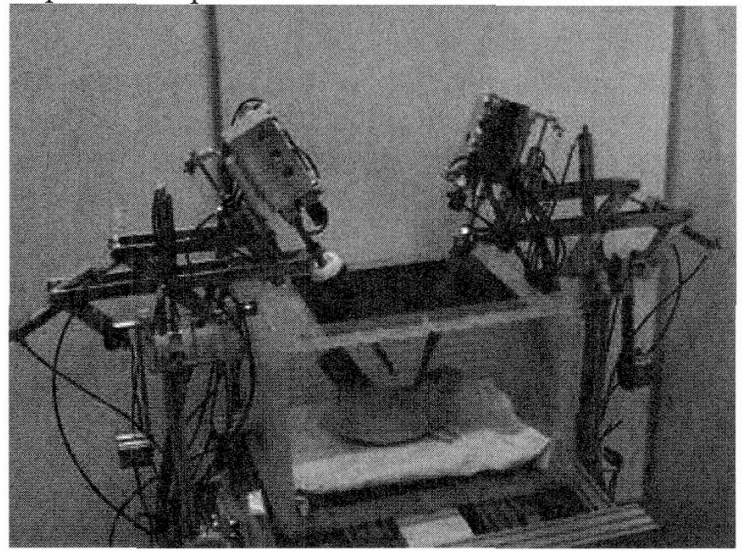

Figure.1 Developed slave manipulator sterilize easy. The measured pressures were used to control the driving force. Position was measured by an encoder having a resolution of $1000 \mathrm{Pulse} / \mathrm{Rev}$. We have confirmed in the preliminary experiment that the position error of the actuator is less than $0.1 \mathrm{~mm}$ using a PID controller.

\section{COMPENSATION FOR PNEUMATIC PIPE LINE}

There are $1 \mathrm{~m}$ length pipelines between the actuators and the servo valves. The inner diameters of the pipelines are $2.5 \mathrm{~mm}$. The pipelines might cause the time delays. However, the pressure sensors should not be installed closer to the actuator to make the forceps easy to sterilize. Some compensation should be better applied. Therefore, pressures at two rooms of the actuators $P_{1}$ and $P_{2}$ and that of at the downstream of the servo valve $P_{1}{ }^{\prime}$ and $P_{2}$ ' were measured in advance during force control. Sinusoidal force waves were given to the actuator by fixed the stroke at middle. Three kinds of amplitudes 1, 5 and $10 \mathrm{~N}$ of force were given with several frequencies.

Fig.2 shows the bode diagrams summarized the experimental results. It became clear that the response between the pressure at the servo valve and that of at the pneumatic actuator could be modeled by a second order delay system given as

$$
\begin{aligned}
& F=\frac{\omega_{n}{ }^{2}}{s^{2}+2 \zeta \omega_{n} s+\omega_{n}{ }^{2}} F^{\prime} \\
& \omega_{n}=28[\mathrm{~Hz}], \quad \zeta=0.6
\end{aligned}
$$

The curves given by Eq.(2) are shown in solid lines in Fig.2. As a result, the driving force at the actuator could be estimated from the pressure difference near the servo valve. As a result, a controller as shown in Fig. 3 was proposed and applied to the force control.

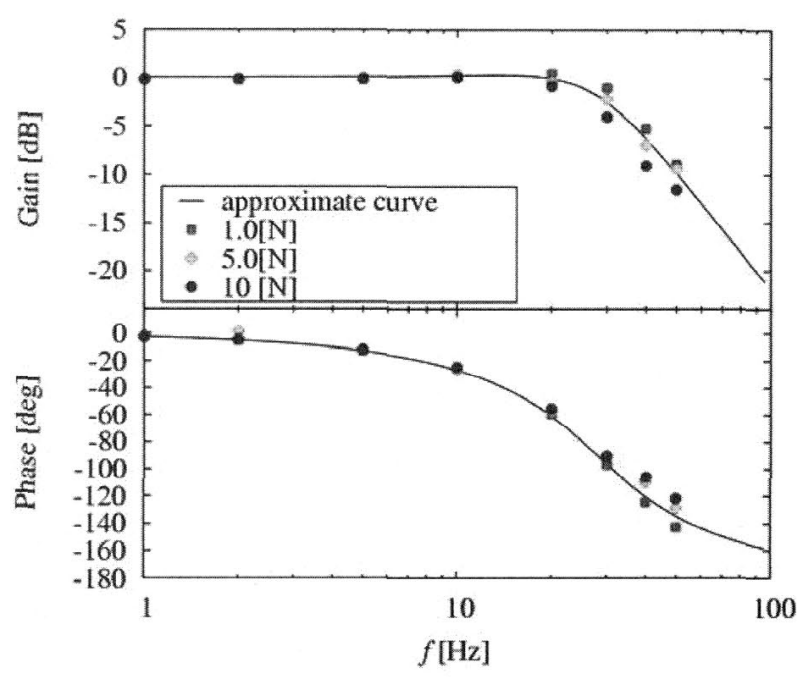

Figure. 2 Bode diagram of pressure responses between $1 \mathrm{~m}$ length pipeline with the diameter of $2.5 \mathrm{~mm}$ 


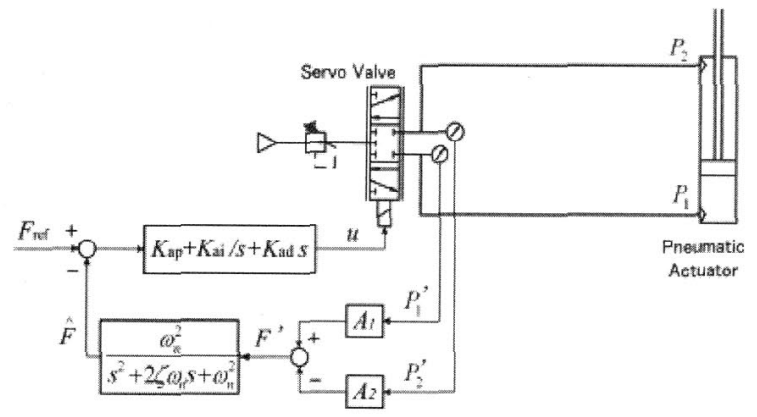

Figure.3 Block diagram of control for driving force

\section{IMPEDANCE CONTROL}

The impedance control is classified into force control type and motion control type. For pneumatic manipulators, the impedance control based on force control is considered to be suitable because of high back drivability and flexible characteristics. Therefore, a torque base impedance control without using force sensors is applied. The reference force at the end effector was given as:

$$
\mathbf{f}_{\text {ref }}=K\left(\mathbf{r}_{\text {ref }}-\mathbf{r}\right)-B \dot{\mathbf{r}}-M \dot{\mathbf{r}}
$$

where,

$\mathbf{r}_{\mathrm{ref}} \in \mathrm{R}^{6}$ : Reference position at Cartesian coordinate

$\mathbf{r} \in \mathrm{R}^{6}:$ Slave position at Cartesian coordinate

$K \in \mathrm{R}:$ Stiffness of the slave manipulator

$B \in \mathrm{R}$ : Viscous coefficient in the slave manipulator $M \in \mathrm{R}:$ Mass

Therefore, the reference torque vector and force vector for joints can be given by

$$
\begin{aligned}
& \boldsymbol{\tau}_{\text {ref }}=J^{T} \mathbf{f}_{\text {ref }}+Z\left(\mathbf{q}, \dot{\mathbf{q}}_{\text {ref }}, \ddot{\mathbf{q}}_{\text {ref }}\right) \\
& \mathbf{F}_{\text {ref }}=J \boldsymbol{\tau}_{\text {ref }}
\end{aligned}
$$

where

$\tau_{\text {ref }} \in \mathrm{R}^{6}:$ Reference torque by the actuator at each joint $\mathrm{Z} \in \mathrm{R}^{6}$ : Inverse dynamics function of the manipulator $q \in \mathrm{R}^{6}:$ Displacement of each joint

$J \in \mathrm{R}^{6 \times 6}$ : Jacobian matrix of the slave manipulator.

The reference velocity and acceleration were obtained from the reference displacement. They were used to calculate the inverse dynamics instead of the velocity and acceleration calculated from the slave position because they include noises. The controller described above can be summarized in a block diagram as shown in the upper part of Fig.4. The compensator for pipeline is implemented at the force controller.

Experiments were conducted with $\mathrm{M}=0.1 \mathrm{~kg}$, $\mathrm{B}=0.02 \mathrm{Ns} / \mathrm{mm}$, and $\mathrm{K}=0.4 \mathrm{~N} / \mathrm{mm}$. The experimental scenery is shown in Fig.5. Gripped a 6 axes force sensor
(BL AUTOTEC.LTD., NANO2.5/2) with the forceps to compare the forces.

The experimental results are shown in Fig.6 when the sinusoidal reference position input was given. It is clear as the controlled force given in Eq.(3) agree well with the measured results by the force sensor, the impedance control was successfully performed.

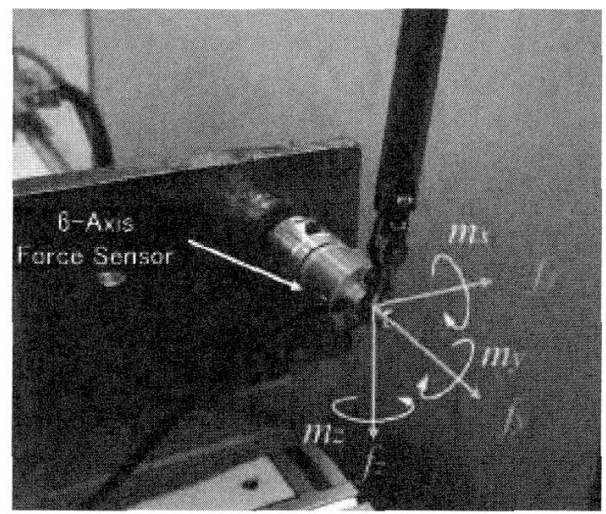

Figure.4 Experimental scenery of impedance control

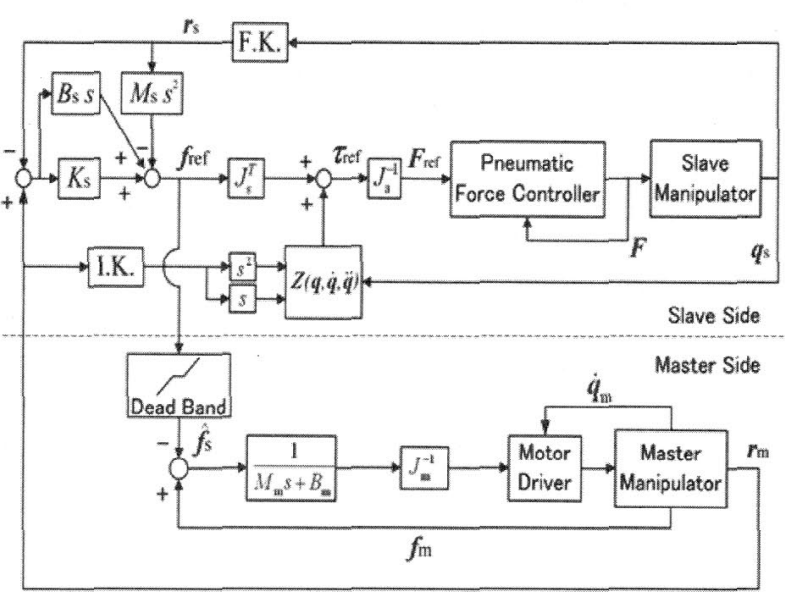

Figure 5 Block diagram

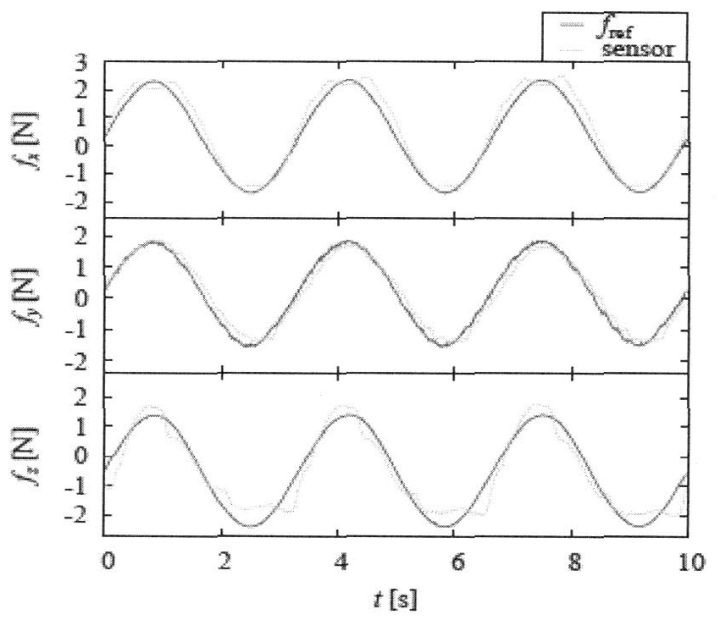

(a) Tranalation

Figure 6 Experimental results of impedance control 


\section{BILATERAL CONTROL}

\section{Master Manipulator}

The master manipulator we have developed used a delta mechanism for translation. In order to realize wide range of the orientation, we mounted a serial gimbal mechanism whose three rotational axes intersect at one point [5][6].

The dynamics of the master manipulator has a high bandwidth with using AC motors with reduction gears. As a result, a motion-based impedance control is suitable, because the dynamics of the manipulator and the effect of the gravity could be compensated by motion control loop and also it is easy to keep the position when the operator released one's hand. Therefore, we applied an admittance control. The block diagram of the controller is summarized in the lower part of Fig. 5 .

\section{Bilateral Control Experiment}

The impedance control at the slave side and the admittance control at the master side shown above were combined for bilateral control (Fig.5). The reference signals become the position and force of the other side. As the force signal from the slave to the master includes some noises, a dead band filter was implemented.

The experimental procedures are as follows: The operator handles the master manipulator to grasp a 6-axes force sensor with the slave manipulator. Then, the operator moves the master manipulator after grasped the sensor as if he or she can feel the force. Experiments were conducted with several impedance and admittance. Experimental results are shown in Fig.7. The impedance at the slave side was given as $\mathrm{M}=0.1 \mathrm{~kg}, \mathrm{~B}=0.02 \mathrm{Ns} / \mathrm{mm}$, and $\mathrm{K}=0.4 \mathrm{~N} / \mathrm{mm}$ and $\mathrm{M}=0.4 \mathrm{~kg}, \mathrm{~B}=0.005 \mathrm{Ns} / \mathrm{mm}$ was given for the master side.

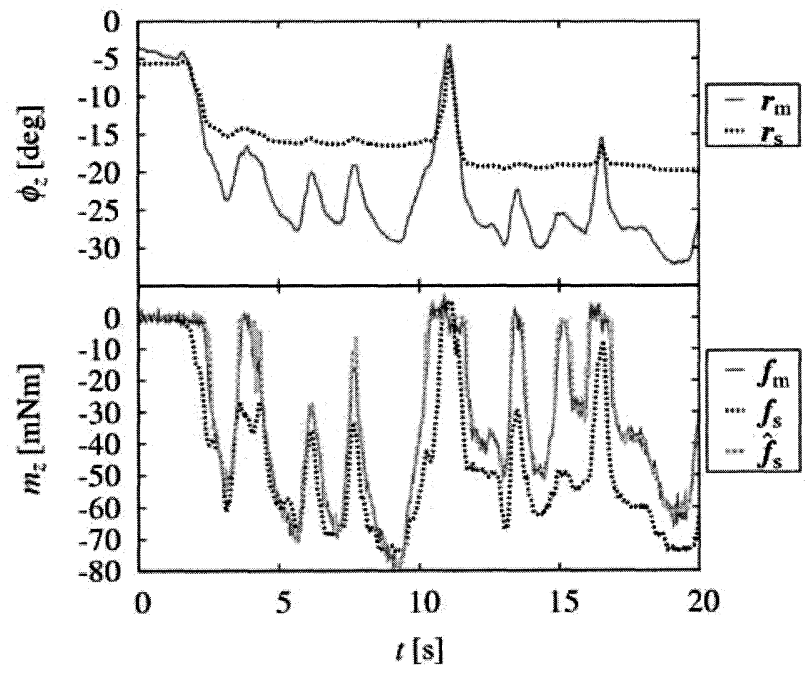

Figure.7 Experimental results of bilateral control
In Fig.7, the horizontal axis shows the time. The upper figure shows the rotational position data around $\mathrm{z}$ axis and the lower figures show the force. The dead band filer was selected to cut off less than $3 \mathrm{~N}$ force and $20 \mathrm{mNm}$ torque because noises can be observed within that range.

It is clear that the control is successfully achieved since the lower figure in Fig.7 shows good agreement. To display more small force would be the next study.

\section{CONCLUSIONS}

In this paper, we developed a master-slave system having 7-DOFs for laparoscopic surgery, which can provide force feedback to the surgeon without using force sensors at the slave manipulator. Pneumatic actuators were used for the slave manipulation because they are effective for a haptic device due to the facility in measurement and control of their driving force.

A control method to compensate the effect of the pipeline between the control valves and the actuators was proposed and applied. Then, an impedance control was applied to the slave manipulator and an admittance control was applied for the master manipulator we have developed. Finally, a bilateral control was achieved combined the controllers. Experimental results showed that the developed system successfully display the contact force on the slave side to the operator on the master side.

\section{REFERENCES}

1. Russell H. Taylor and Dan Stioianovici, Medical Robotics in Computer-Integrated Surgery, IEEE Trans. on Robotics and Automation, 2003, 19-5,pp.765-780

2. G.H. Ballantyne, J. Marescaux, and P.C.Giulianotti, editors. Primer of Robotic \& Telerobotic Surgery. Lipincott Williams \& Wilkins, 2004

3. G.S.Guthart and J.K.Salisbury, The intuitive telesurgery system: Overview and application, Proc. of IEEE ICRA, 2000, pp.618-621

4. O. Gerovichev, P. Marayong, A.M. Okamura, The Effect of Visual and Haptic Feedback on Computer-Assisted Needle Insertion, Computer -Aided Surgery, 2004, 9-6,pp.243-249

5. K.Tadano and K.Kawashima, Development of a Master Slave Manipulator with Force Display using Pneumatic Servo System for Laparoscopic Surgery, International Journal of Assistive Robotics and Mechantronics, 2007, 8-4,pp.6-13

6. K. Tadano, K. Kawashima and T. Kagawa,Evaluation of a Master Slave System with Force Sensing, Proc. of SICE Annual Conference, 2007, pp.2871-2874 